\title{
GROUPOÏDES AUTOMORPHES PAR LE GROUPE GYGLIQUE
}

\author{
A. SADE
}

\section{Introduction}

1. Définitions. Nous appellerons groupoïde un ensemble non vide, $G$, muni d'une loi $(\times)$ faisant correspondre à tout couple ordonné $x, y \in G$, au plus un élément $z$ de $G$, appelé produit de $x$ par $y$, et satisfaisant à la loi d'homogénéité (2). Si $E$ est l'ensemble des $z$ la loi $(X)$ définit une application de $E$ sur $G$. On dira que $G$ est incomplet si $E \subset G G$ et ordinaire si $E=G G$.

Un semi-groupe (5) est un groupoïde associatif.

Un quasigroupe, ordinaire ou incomplet, est un groupoïde dont les éléments ont au plus un quotient à droite et un quotient à gauche. Le mot "diviseur" sera entendu au sens de "sous-quasigroupe".

Soit $Q$ un groupe additif d'entiers, ou d'entiers mod. $n$, et $T$ un sous-groupe de $Q$. Soit $(X)$ une loi binaire des éléments de $Q$ telle que:

(1) $Q$ soit un groupoïde,

(2) l'application $x \rightarrow x+t, t \in T$, soit un automorphisme de $Q$. La structure ainsi définie est appelée un groupoïde automorphe par le groupe cyclique $T$ et est désignée par $Q(\times)$. Si $Q=T$ on dira que $Q(X)$ est automorphe par le groupe cyclique.

2. Exemple I. L'ensemble des nombres rationnels muni de la loi:

$$
x \times y=\frac{1}{2}(x+y)+C
$$

3. Exemple II. Le quasigroupe:

$$
x \times y=-(x-y-1)^{4}+4(x-y-1)+y+5 \quad(\bmod .7)
$$

4. Un groupoïde $G(X)$ automorphe par le groupe cyclique est entièrement défini par la fonction $f(x)=x \times 0$, ou par l'application

$$
S: \quad x \rightarrow x S=f(x) \text {, }
$$

d'un certain sous-ensemble de $G$, dans $G$; la fonction $f(x)$ étant définie pour tout élément de ce sous-ensemble.

5. La condition exprimant qu'un groupoïde, automorphe par le groupe cyclique, est un semi-groupe n'est pas simple. Bornons-nous à ce résultat: Il n'existe aucun groupe automorphe par le groupe cyclique.

Reçu le 7 avril, 1956. 
6. Pour qu'un groupoüde $G(X)$, automorphe par le groupe cyclique, soit un quasigroupe il faut et il suffit que la fonction $x \times 0=f(x)$ satisfasse aux deux conditions :

$$
\left\{\begin{array}{l}
\text { (i) } f(x)=f(y) \rightleftarrows x=y \\
\text { (ii) } x-f(x)=y-f(y) \rightleftarrows x=y
\end{array}\right.
$$

(mod. $n$ si $G$ est fini et d'ordre $n$.)

La première exprime la loi de cancellation à droite $\left(\mathrm{N}^{0} 1\right)$ pour $y=0$. Montrons qu'elle est obéie par deux facteurs quelconques. L'égalité:

$$
x \times a=y \times a,
$$

s'écrit, en vertu de l'automorphișe:

Donc (i)

$$
\begin{aligned}
(x-a) \times 0+a & =(y-a) \times 0+a \\
f(x-a) & =f(y-a)
\end{aligned}
$$

(mod. $n$ si $G$ est fini.)

La seconde exprime la loi de cancellation à gauche.

Les réciproques! sont immédiates.

7. Les quasigroupes infinis, automorphes par le groupe cyclique, ont peu de propriétés. Toute fonction $f(x)$ ayant une dérivée toujours négative, ou toujours plus grande que un, ou toujours eomprise entre 0 et 1 , fournit une solution.

8. Tout quasigroupe ordinaire fini, automorphe par le groupe cyclique, est d'ordre impair.

Le quasigroupe étant ordinaire, $f(x)$ décrit toutes les valeurs de $x$; donc, en désignant par $n$ l'ordre du quasigroupe:

$$
\Sigma f(x)=0+1+2+\ldots+(n-1)=S \quad \text { (mod. } n) \text {. }
$$

D'après (ii), $N^{\circ} 6$, la fonction $x-f(x)$ décrit ce même ensemble, donc:

$$
\begin{aligned}
& \geq[x-f(x)]=S=S-S \\
& S=n(n-1) / 2 \equiv 0
\end{aligned}
$$

$(\bmod . n)$, $(\bmod . n)$

$n=2 M+1$.

Mais il existe des quasigroupes d'ordre pair incomplets, automorphes par le groupe cyclique.

$$
\begin{aligned}
& \text { Exemples: } \quad x=0123 \text {; } \quad \text { et } \quad x=012345 \\
& f(x)=20-3 \quad f(x)=542-03 .
\end{aligned}
$$

Le quasigroupe incomplet du $6^{\text {ième }}$ ordre: $x=012345 ; x \times 0=301425$; $x \times 1=152-0-; x \times 2=415230 ; x \times 3=2-314-; x \times 4=$ $520314 ; x \times 5=0-4-53$, est automorphe par le sous-groupe du $3^{\mathrm{e}}$ ordre du groupe cyclique:

$$
T=024.135 ; T^{2} ; T^{3}=1 .
$$


Il existe un quasigroupe ordinaire, automorphe par $x \rightarrow x+i,(i=0,3,6)$ et défini par:

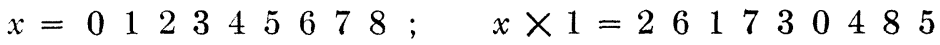

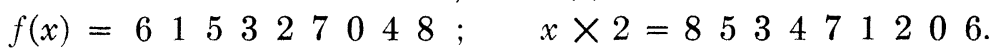

\section{Transformations préservant l'invariance par le groupe cyclique.}

9. D'après le $\mathrm{N}^{0} 4$ on peut identifier tout quasigroupe automorphe par. le groupe cyclique avec la fonction correspondante:

$$
x \times 0=f(x)
$$

et parler du quasigroupe $f(x) \bmod$. $n$. On peut même le représenter par la substitution $x \rightarrow f(x)$ mise sous la forme de produit de cycles.

10. Si $f(x)$ définit un quasigroupe automorphe par le groupe cyclique, les fonctions:

$$
\begin{array}{cl}
a: & x-f(x), \\
b: & -f(-x), \\
a b=\dot{b} a: & x+f(-x) \\
c \vdots: & f^{-1}(x) \\
D_{h}: & f(x+h), \\
E_{h}: & f(x)+h,
\end{array}
$$
(réciproque),

jouissent de la même propriété.

Soit $f(x)$ une fonction satisfaisant aux conditions (3) du $\mathrm{N}^{\circ} 6$. Alors,

$$
g(x)=x-f(x)
$$

sera encore une solution, car

$$
g(x)=g(x) \rightleftarrows x-f(x)=y-f(x) \rightleftarrows x=y,
$$

puisque $f(x)$ est une solution.

$$
x-g(x)=f(x),
$$

donc

$$
x-g(x)=y-g(y) \rightleftarrows f(x)=f(y) \rightleftarrows x=y .
$$

Les autres parties s'établissent de manière analogue.

Si $f(x)$ est d'ordre $n$, les $n$ opérations $E_{h},(h=0,1, \ldots, n-1)$ forment un groupe cyclique car, si l'on désigne chaque opération par la valeur correspondante de $h$, ce groupe est isomorphe au groupe additif des restes, mod. $n$.

11. Groupes formés par les opérations précédentes. Considérons l'ensemble $F$ de toutes les fonctions $f(x)$ solutions des conditions (3) pour une valeur donnée de $n$. Chacune de ces opérations $a, b, c, D, E$ fait correspondre à chaque $f(x)$ une $f(x)$ et une seule et réciproquement. Ce sont des transformations de F. Elles forment un groupe, sous-groupe du groupe total (8, p. 3). 
Les opérations $a, b$ et les opérations $b, c$ engendrent deux groupes carrés. En effet, $a b=b a\left(\mathrm{~N}^{\circ} 10\right)$, et $b c=c b$, car l'une comme l'autre transforme $x \rightarrow$ $f(x)$ en $x \rightarrow-f^{-1}(-x)$.

L'opération ca $=(a c)^{-1}$ est du $6^{\text {ème }}$ ordre et $(c a)^{3}=b$.

Les opérations $a, b, c$ engendrent un groupe diédral du $12^{\mathrm{me}}$ ordre, défini par:

$$
a b=b a, b c=c b, a^{2}=b^{2}=c^{2}=1,(a c)^{3}=(c a)^{3}=b .
$$

12. Les opérations $E_{h}$ et $D_{h}$ sont transformées l'une de l'autre par $c$.

$$
c^{-1} E_{h} c=D_{h} \text {. }
$$

13. $D$ et $E$ sont des isotopies (1). Si $D_{h}=E_{k}$, en posant $f(x+h)=z$ et $f(x)=y$, on aura:

et

$$
f(x+h) E_{k}=z+k
$$

$f(x+h) D_{h}=y$,

d'où :

ou:

et enfin

$$
y=z+k
$$

$$
f(x)=f(x+h)+k
$$

$$
f(x)=a x+b \quad(a h+k \equiv 0, \bmod . n) .
$$

Pour que $f(x)$ et $x-f(x)$ décrivent toutes les valeurs de $x$ il faut de plus que $a$ et $a-1$ soient premiers avec $n$. Ainsi:

Les quasigroupes $f(x)$ pour lesquels toute opération $D$ est aussi une opération $E$ sont $f(x)=a x+b$, où $a$ et $a-1$ sont premiers avec $n$.

Les opérations $(a),(b),(c)$ du $\mathrm{N}^{0} 10$ laissent cette propriété invariante.

Si $a=(n+1) / 2$, on aura un quasigroupe abélien.

14. Voici les lois de composition de tous les quasigroupes ordinaires, automorphes par le groupe cyclique, jusqu'à $n=9$. $a$ et $a-1$ sont premiers avec $n, b$ et $c$ sont quelconques.

Pour toute valeur de $n$ on a d'abord la solution:

$$
x \times y=a(x-y)+y+b
$$

Pour $n=7$, on a, en plus:

$$
x \times y= \pm(x-y+c)^{4}+4(x-y+c)+y+b .
$$

Pour $n=9$, on trouve 28 solutions, aux isotopies $D$ et $E$ près, dont

$$
x \times y=3(x-y)^{2}+2 x-y
$$

et

$$
x \times 0=1+6\left(\begin{array}{l}
x \\
1
\end{array}\right)+7\left(\begin{array}{l}
x \\
2
\end{array}\right)+2\left(\begin{array}{l}
x \\
3
\end{array}\right)+5\left(\begin{array}{l}
x \\
5
\end{array}\right)+3\left(\begin{array}{l}
x \\
6
\end{array}\right)+6\left(\begin{array}{l}
x \\
7
\end{array}\right) .
$$


Quand $n$ est pair, il faut avoir recours à des fonctions irrationnelles. Exemple, $n=4$ :

$$
f(x)=x \times 0=\sqrt{\left(17 x^{2}-41 x+24\right) / 6}
$$

représente le quasigroupe du $\mathrm{N}^{0} 8$.

\section{Composition des quasigroupes}

15. Si $f(x)$ est un quasigroupe d'ordre p automorphe par le groupe cyclique et si $\rho_{x}(y), x=0,1, \ldots, p-1$, sont $p$ quasigroupes d'ordre $m$ encore automorphes par le groupe cyclique, alors:

$$
F(x+p y)=f(x)+p \rho_{x}(y)
$$

$(\bmod . n)$

est un quasigroupe d'ordre $n=m p$, automorphe par le groupe cyclique.

$$
\begin{aligned}
& \text { Exemple: } f=(01)(2) ; \rho_{0}=(0214)(3) ; \rho_{1}=(02)(34)(1) ; \rho_{2}=(0412)(3) . \\
& \begin{array}{rrrrrrrrrrrrrrrr}
X \\
X=x+3 y= & 0 & 1 & 2 & 3 & 4 & 5 & 6 & 7 & 8 & 9 & 10 & 11 & 12 & 13 & 14 \\
F(X)= & 7 & 6 & 14 & 13 & 3 & 8 & 4 & 0 & 2 & 10 & 12 & 11 & 1 & 9 & 5
\end{array}
\end{aligned}
$$

Quand $x$ décrit le champ $(0, p-1)$ et y le champ $(0, m-1)$, la fonction $f^{\prime}(x)$ d'une part et chacune des fonctions $\rho_{x}(y)$ de l'autre décrivent respectivement les mêmes champs. Donc $F$ prend toutes les valeurs de 0 à mp -1 et il en est de même de $x-F(x)$.

16. Dans les conditions $d u \mathrm{~N}^{0} 15$, il existe une partition régulière sur le quasigroupe $F(X)$. Les $p$ cosets sont les ensembles $x+p y$, où $y$ décrit les $m$ valeurs $0,1, \ldots, m-1$. A chaque valeur de $x$ correspond un coset; le système des représentants est $x=0,1, \ldots, p-1$. Le quasigroupe quotient est isomorphe au quasigroupe $f(x)$. Pour que la partition définisse un diviseur normal (4) il faut et il suffit que $f(x)$ soit idempotent; tous les cosets sont alors diviseurs nor$\operatorname{maux}$.

Il est d'abord évident que les ensembles $x+p y$ sont disjoints car si

$$
a+p y \equiv b+p y^{\prime} \quad(\bmod . m p) \text {, }
$$

on aura

$$
a-b \equiv 0 \quad(\bmod . p),
$$

donc

$$
a=b,
$$

puisque $a$ et $b$ sont plus petits que $p$.

Soient $a+p y$ un élément du coset $x=a$ et $b+p y^{\prime}$ un élément du coset $x=b$. En notant $(X),(*)$ et $(\cdot)$ les opérations de $f, \rho_{a-b}$ et $F=f+p \rho$, leur produit sera: 


$$
\begin{aligned}
P=(a+p y):\left(b+p y^{\prime}\right) & =\left[a-b+p\left(y-y^{\prime}\right)\right] \cdot 0+b+p y^{\prime} \\
& =F\left[a-b+p\left(y-y^{\prime}\right)\right]+b+p y^{\prime} \\
& =f(a-b)+p \rho_{a-b}\left(y-y^{\prime}\right)+b+p y^{\prime} \\
& =(a-b) \times 0+b+p\left[\left(y-y^{\prime}\right) * 0+y^{\prime}\right] \\
P & =a \times b+p\left(y * y^{\prime}\right)=c+p y^{\prime \prime} .
\end{aligned}
$$

Ainsi $P$ appartient au coset représenté par $x=a \times b=c$.

La loi de composition des cosets est la même que celle de leurs représentants èt il existe un homomorphisme canonique de $F$ sur $f$.

Si $f$ n'a aucun élément idempotent, aucun coset n'est fermé. Si un coset est diviseur normal, tous le seront; car si $a \times a=a$, tous les éléments de $f(x)$ seront idempotents, à cause de l'automorphisme $(a+1) \times(a+1)=a+1$.

Exemple. Lè quasigroupe examiné au $\mathrm{N}^{0} 15$ et représenté, avec la notation du $N^{\circ} 9$, par:

$$
F(x)=(0,7)(1,6,4,3 ; 13,9 ; 10,12)(2,14,5,8)
$$

a trois cosets

$$
0,3,6,9,12 ; 1,4,7,10,13 ; \text { et } 2,5,8,11,14 \text {, }
$$

mais $f(x)$ h'ayaht aucun élément idempotent, aucun coset n'est diviseur normal; il y a seulement partition régulière et homomorphisme naturel $\mathrm{du}$ quasigroupe $F$ sur le quasigroupe quotient, isomorphe à $f$.

Soit au contraire le quasigroupe défini par:

$$
p=3 ; \mathrm{m}=5 ; f(x)=(12)(0) ; \dot{\rho}_{0}=\rho_{1}=\rho_{2}=(0)(1243) ;
$$

d'où :

$$
F=(1,2)(3,6,12,9)(4 ; 8,13,11)(5 ; 7,14 ; 10)(0)
$$

(Notation du No 9 ).

Comme $f(x)$ ešt idempotent, tous les cosets sont diviseurs normaux.

17. Si $\rho_{x}(y)$ ne dépend plus de $x$, et si l'on considère, pour l'ensemble de tous les nombres entiers $(p=1,2,3, \ldots$,$) tous les quasigroupes f(x)$ (mod. $p$ ) automorphes par le groupe cyclique d'ordre $p$, les fonctions $f$ forment un semi-groupe (5) non commutatif, avec unité bilatère, par rapport à la loi de composition

$$
f * \rho=f+p \rho .
$$

Cet ensemble est fermé d'aprè̀ le $\mathrm{N}^{\circ} 15$. Il est associatif car, si $f, \rho$ et $\psi$ sont trois fonctions, mod. $p, q$ et $r$ respectivement, on a

$$
\begin{aligned}
& (f * \rho) * \psi=(f+p \rho) * \psi=f+p \rho+p q \psi \\
& \text { (mod. pqr), } \\
& f *(\rho * \psi)=f *(\rho+q \psi)=f+p \rho+p q \psi
\end{aligned}
$$

L'unité est la fonction $f=0$, correspondant au quasigroupe du $1^{\text {er }}$ ordre $0 * 0=0=u$; elle est bilatère car $f * u=u * f=f$ : 
18. Si $p, p^{\prime}, \ldots$ sont des entiers positifs quelconques et si $M=\left\{p, p^{\prime}, \ldots\right\}$ est le semi-groupe multiplicatif engendré par ces entiers, l'ensemble des fonctions $f(x)$ (mod. $q), q \in M$, est un semi-groupe, diviseur du semi-groupe défini au $N^{\circ}$ précédent.

Car il est fermé et contient l'unité; il est associatif. En particulier l'ensemble, au sens de la multiplication, $*$, définie au $\mathrm{N}^{0} 17$, des puissances d'une fonction unique $f(x)$, d'ordre $q$, est isomorphe au semi-groupe additif des entiers. Si l'on pose:

$$
f^{* \mathbf{p}}=0+f+q f+q^{2} f+\ldots+q^{\mathrm{p}-1} f,
$$

alors,

$$
f^{* \mathrm{p}} * f^{* \mathrm{r}}=f^{*(\mathrm{p}+\mathrm{r})}
$$

\section{Recherches sur l'automorphe $(8, p .40)$ de $f(x)$}

19. Quand un quasigroupe $Q(\times)$, fini ou non; automorphe par toutes les transformations $C ; x \rightarrow x+h$, est engendré par un de ses éléments, son automorphe se réduit au seul groupe cyclique $\hat{C}$.

Soit $Q=\{a\}$; à cause de l'automorphisme $x \rightarrow x-a, Q$ est aussi engendré par l'élément zéro. Soit

$$
\left(\begin{array}{l}
x \\
x^{\prime}
\end{array}\right)=H
$$

un automorphisme quelconque de $Q$, et $v$ l'image de 0 par $H$. On montre par induction que, pour tout $x$ dans $Q$, on aura: $x H=x^{\prime}=x+v$.

Soient $a$ et $b$ deux élément de $Q$ pour lesquels on suppose:

$$
a^{\prime}=a+v \text { et } b^{\prime}=b+v .
$$

En vertu de l'automorphisme $C$ :

$$
a^{\prime} \times b^{\prime}=\left(a^{\prime}-b^{\prime}\right) \times 0+b^{\prime}=(a-b) \times 0+b+v,
$$

et comme

$$
\begin{gathered}
a \times b=(a-b) \times 0+b, \\
a^{\prime} \times b^{\prime}=a \times b+v .
\end{gathered}
$$

La propriété étant vraie pour $a=b=0$; et $Q$ étant engendré par 0 , la propriété est générale et $H \in C$.

20. En particulier, si l'une des trois équations: $0 \times 0=x ; x \times 0=0$; $0 \times x=0$ a pour solution un nombre premier avec $n$, le quasigroupe $Q(X)$, d'ordre n, automorphe par le groupe cyclique $C$, admet $C$ pour automorphe. 
21. Si un quasigroupe $Q(\times)$ est automorphe par le groupe cyclique, si $0 \times 0=a$ et si $x \rightarrow x^{\prime}$ est un automorphisme quelconque de $Q$, on a, pour toute valeur de $k$;

$$
(k a+r)^{\prime}=k a+r^{\prime} .
$$

La proposition est évidente si $k$ est nul et on la généralise sans peine par induction.

22. Si $Q(X)$ est un quasigroupe d'ordre $n$, automorphe par le groupe cyclique $C=\{c\}, c=0,1, \ldots, n-1$, tout diviseur propre $D$ de $Q$ est composé des éléments de une ou plusieurs suites;

$$
i, i+p, i+2 p, \ldots, i+k p, \ldots, i+n-p,
$$

où $p \mid n$ et $i<p$; la valeur de $p$ restant la même pour toutes les suites de $D$. Il existe $p$ telles suites, disjointes, correspondant aux $p$ valeurs de $i(i=0,1, \ldots$, $p-1)$. Enfin, $D$ est automorphe par le diviseur $\left\{c^{p}\right\}$ du groupe cyclique $C$.

Puisque $C$ opère transitivement sur $Q$, tout élément de $C$ ne peut pas être un automorphisme de $D$. Comme les conditions $c^{\alpha} D=D \mathrm{c}^{\beta} D=D$ impliquent que $c^{\alpha-\beta} D=D$, le sous-ensemble des éléments de $C$, qui sont des automorphismes de $D$, forme un sous-groupe. $C$ étant cyclique, ce sousgroupe est de la forme $\left\{c^{p}\right\}$, où $p \mid n$ et est le plus petit entier satisfaisant à $c^{p} D=D$

Si $D$ contient l'élément i, il contiendra les $n / p=m$ éléments distincts:

$$
i, i+p, i+2 p, \ldots, i+k p, \ldots, i+n-p .
$$

L'automorphisme $x \rightarrow x+r,(r<p)$ transformera $D$ en un diviseur $D^{\prime}$, pouvant coincïder avec $D$, qui contiendra les éléments:

$$
i+r, p+i+r, \ldots, k p+i+r, \ldots, n-p+i+r .
$$

On voit immédiatement que les suites (5) correspondant à des valeurs distinctes de $r$ sont disjointes. Elles réalisent une partition des $n$ éléments de $Q$, épuisant l'ensemble de ces éléments, puisqu'elles sont disjointes, au nombre de $p$, et composées de $n / p$ éléments chacune.

23. Si un quasigroupe $Q(\times)$ d'ordre $n$, automorphe par le groupe cyclique, $x \rightarrow x+h$, n'est engendré par aucun de ses éléments.

(1) Chaque élément de $Q$ engendre un diviseur d'ordre constant $m(m p=n)$.

(2) Le nombre des diviseurs distincts ainsi définis est p et chacun d'eux est engendré par n'importe quel de ses éléments.

(3) Ces p diviseurs sont disjoints, isomorphes entre eux et l'on passe de l'un à tous les autres par les transformations:

$$
x \rightarrow x+h(h=0,1,2, \ldots, p-1) .
$$


(4) Le diviseur engendré par l'élément 0 est: $D=0, p, 2 p, 3 p, \ldots, n-p$, et on l'obtient en multipliant par $p$, sans changer la loi de composition, un quasigroupe quelconque d'ordre $m$, automorphe par le seul groupe cyclique d'ordre $m$, c'est-à-dire engendré par un seul de ses éléments.

(5) Tous ces diviseurs ont le méme automorphe, à savoir le groupe cyclique d'ordre $m$ :

$$
x \rightarrow x+\mathrm{k} p, k=0,1,2, \ldots, m-1 \text {. }
$$

Preuve. Soit un quasigroupe $Q(X)$ d'ordre $n$, non monogène, et automorphe par le groupe cyclique $C: x \rightarrow x+h ; h=0,1,2, \ldots, n-1$. Soit $D$ le sousquasigroupe propre engendré par l'élément zéro: $\{0\}=D \subset Q$. Si l'on fait subir à $D$ toutes les transformations $C$, à chaque valeur $i$ de $h$ correspondra un diviseur $D_{i}$, isomorphe à $D$ :

$$
D_{i}=D\left(\begin{array}{c}
x \\
x+i
\end{array}\right)
$$

et $D_{i}$ sera engendré par l'élément $i$ de la même manière que $D$ l'était par l'élément 0 . On obtiendra ainsi $n$ diviseurs.

Soit $a$ un élément non nul de $D, D_{a}=\{a\}$. Comme $a \in D, D_{a} \subset D$. Mais $D_{a} \cong D$, donc $D_{a}=D$. Ainsi chacun des $n$ diviseurs $D_{i}$ est engendré par n'importe quel de ses éléments.

Soit $p$ le plus petit élément non nul de $D=\{0\}$. Donc

$$
D_{p}=\{p\}=D=D\left(\begin{array}{c}
x \\
x+p
\end{array}\right)
$$

Par suite $D$ contient, outre l'élément zéro, les éléments $p, 2 p, 3 p, \ldots, k p ; \ldots$ $(\bmod . n)$, dont le plus petit $(\bmod . n)$ est $p$ par hypothèse. On voit facilement que $p \mid n$ et $k p<n$; on peut poser $m p=n ; k<m$. Supposons alors que $D$, en plus de $0, p, 2 p, \ldots,(m-1) p$, contienne un autre élément $q$, non multiple de $p$, par exemple:

$$
k p<q<k p+p \leqslant n
$$

l'égalité correspondant au cas où $k=m-1$.

S'il en était ainsi, le diviseur:

$$
D^{\prime}=D\left(\begin{array}{c}
x \\
x-k p
\end{array}\right)
$$

contiendrait l'élément zéro et coïnciderait avec $D$. D'autre part, il contiendrait l'élément $q-k p$. Or

$$
0<q-k p<p
$$

et, contrairement à l'hypothèse, $p$ ne serait pas le plus petit élément non nul de $D$. Ainsi, $D=0, p, 2 p, \ldots, k p, \ldots,(m-1) p$.

Chaque diviseur $D_{i}$ est d'ordre $m$ et le nombre des $D_{i}$ distincts est $p$. 
D'après le $\mathrm{N}^{0}$ précédent ces diviseurs sont disjoints. Si l'on remplace dans $D$ tous les éléments $0, p, 2 p, \ldots, n-p$ par leurs quotients par $p$ :

$$
0,1,2, \ldots, m-1
$$

en respectant la loi de composition de $Q$, on obtient évidenment un quasigroupe isomorphe à $D$ par $x \rightarrow(x / p)$, dont l'automorphe se réduit $\left(\mathbb{N}^{\circ} 19\right)$ au groupe cyclique d'ordre $m: x \rightarrow x+k,(k=0,1,2, \ldots, m-1)$ puisqu'il est engendré par l'un quelconque de ses éléments.

24. Si un quasigroupe $Q(X)$ est automorphe par le groupe cyclique et si $D_{0}, D_{1}, D_{2}, \ldots, D_{p-1}$ sont les diviseurs d'ordre $m=n / p$ engendrés par les divers éléments de $Q$, tout automorphisme $T$ de $Q$ projette chaque diviseur $D_{i}$ sur un diviseur $D_{j}$, l'isomorphisme induit par $T$ entre $D_{i}$ et $D_{j}$ étant:

$$
x \rightarrow x+j-i \quad(i T=j)
$$

L'automorphe de $Q$ induit sur l'ensemble des indices $0,1,2 \ldots, p-1$ un groupe de transformations.

Preuve. Soit $T$ un automorphisme de $Q$ et soit a l'image de zéro.

$$
0 T=a .
$$

Alors tous les produits engendrés par 0 se projettent sur les produits engendrés de la même manière par $a$ et tous les éléments de $D_{0}$ ont des images bieh déterminées et définies par la seule donnée de l'image de 0 .

Si $0 \times 0=k p$, d'après l'automorphisme,

$$
a \times a=k p+a,
$$

de sorte que;

$$
D_{0} T=D_{i} \text {. }
$$

Plus généralement si $i$ a pour image $j$; l'image de $\{i\}$ sera évidenment $\{j\}$; car si

$$
i T=j=i+h,
$$

d'après le $\mathrm{N}^{\circ} 21$

$$
(k p+i) T=k p+i T=k p+i+h, \quad D_{i} T=D_{i+h}=D_{i} .
$$

Tout automorphisme $T$ de $Q$ induit donc sur l'ensemble des diviseurs $D_{n}$; $D_{1}, D_{2}, \ldots, D_{p-1}$ une transformation $t$ des indices $0,1,2,3, \ldots, p-1$. L'automorphe de $Q$ induit sur l'ensemble $0,1,2, \ldots, p-1$ un groupe de substitutions.

25. Dans les conditions $d u N^{\circ} 24$, si g est l'ordre du système minimal dr générateurs de Q, l'ordre de l'automorphe de Q est un diviseur de $m^{g} p ! /(p-g)$ !: 
Si l'on choisit un élément quèlconque dans chaque diviseur: $a_{0} \in D_{0}$, $a_{1} \in D_{1}, \ldots, a_{i} \in D_{i}, \ldots$ d'après le $\mathrm{N}^{\circ} 24$, tout automorphisme $T$ de $Q$ sera entièrement défini si l'on connaît les images:

$$
a_{0}^{\prime}=a_{0} T, \ldots, a_{i}^{\prime}=a_{i} T, \ldots
$$

Mais ces images ne sont pas indépendantes enaénéral.

Soit $a_{i}, a_{j}, \ldots, a_{k}$ un système minimal de générateurs indépendants de $Q$ (c'est-à-dire comprenant le plus petit nombre possible de générateurs tels que, par la suppression d'un de ces générateurs, les éléments restants engendrent seulement une partie propre de $Q$ ). Alors si les images des générateurs sont connues, il est clair que l'image d'un élément quelconque de $Q$ sera définie. Le nombre des éléments de $Q$ dont on peut se donner arbitrairement l'image est donc égal au nombre minimum des générateurs; soit $g$ ce nombre.

On observera encore que ces $g$ générateurs appartiennent nécessairement à $g$ diviseurs distincts parmi les diviseurs $D_{0}, D_{1}, \ldots, D_{p-1}$ Car si deux d'entre eux appartenaient au même diviseur, $D$, on aurait évidemment un système de générateurs plus court en supprimant l'un d'eux.

11 y a

$$
m^{o}\left(\begin{array}{l}
p \\
g
\end{array}\right) g !=m^{g} p ! /(p-g) !=q
$$

manières de choisir les images des $g$ générateurs. Les substitutions ainsi obtenues forment un groupe d'ordre $q$, dont l'automorphe de $Q$ est un diviseur, toutes ces substitutions n'étant pas nécessairement des automorphismes de $Q$.

Si $p=1$, alors $g=1, m=n=q$ et l'automorphe de $Q$ est d'ordre $n$; conformément à la conclusion du $\mathrm{N}^{\circ} 19$.

26: Le diviseur $D_{o}$ ñest pas nécessairemeñt normal.

Exemple.

Le quasigroupe:

$$
S: F(x)=(0,10)(1,3,11,14,8,9, \overrightarrow{7}, 13,12,4)(2,6)(\overline{5})
$$

automorphe par le groupe cyclique du $15^{\circ}$ ordre, a pour diviseurs

$$
D_{i}=i, \tilde{5}+i, 10+i \quad(i=0,1,2,3,4) \text {. }
$$

Mais aucun de ces diviseurs n'est normal.

27: Si $Q$ est un quasigroupe d'ordre $\ddot{n}=m p$, automorphe par le groupe cyclique $C_{n}$, si $D_{0}, D_{1}, \ldots, D_{p-1}$ sont les $\not$ diviseurs d'ordre $m$ engendrés par les divers éléments de $Q$, pour que $D_{0}$ (et par conséquent tout $D_{i}$ ) soit normal dans $Q$ il faut et il suffit que $Q$ soit défini par une fonction $F(i+\dot{p} y)=f(i)+\dot{p} \rho_{i} y$ oì $j=f(i)$ est un quasigroupe idempotent d'ordre $p$, automorphe par le groupe 
cyclique $C_{p}$ et où $\rho_{i}(y),(y=0,1,2 \ldots, m-1)$ définit pour chaque valeur de $i$ $(i=0,1, \ldots, p-1)$ un quasigroupe d'ordre $m$, automorphe par le groupe cyclique $C_{\mathrm{m}}$.

On a alors $D_{i} \times D_{0}=D_{j}$ et le quasigroupe quotient $Q / D$ est isomorphe $\grave{a}$ $f(i)$ par $D_{i} \rightarrow i$.

Preuve. Si $D$ est normal, tout $D_{i}$ est normal et il est évident que, pour toute valeur de $i$, les produits de tous les éléments de $D_{i}$ par 0 doivent appartenir à un seul diviseur, par exemple $D_{j}$. Réciproquement, si cela a lieu, il est facile de montrer que tous les $D_{i}$ sont normaux. En effet, par définition (23), on a:

$$
D_{i}=D_{0}\left(\begin{array}{c}
x \\
x+i
\end{array}\right) .
$$

Supposons: $D_{i} \times 0=D_{j}$, alors, à cause de l'automorphisme

$$
D_{i+k p} \times k p=D_{j+k p} .
$$

Mais $D_{i+k p}=D_{i}$, puisque

$$
D_{i+k p}=D_{i}\left(\begin{array}{c}
x \\
x+k p
\end{array}\right)
$$

et que $D_{i}=i, i+p, \ldots$, donc

$$
D_{i} \times k p=D_{j}
$$

quelque soit $k$, ce qui signifie

$$
D_{i} \times D_{0}=D_{j}
$$

et par suite

$$
D_{i+h} \times D_{h}=D_{j+h} .
$$

Donc $D_{0}$ sera bien normal si, pour toute valeur de $i(i=0,1,2, \ldots, \mathrm{p}-1)$ on a

$$
D_{i} \times 0=D_{j}
$$

Cette relation établit une correspondance biunivoque entre chaque élément de $D_{i}: i+k p \in D_{i}$ et un élément: $j+z_{k} p$ de $D_{j}$. Reste à exprimer que cette transformation satisfait pour tout $x \in Q$, à la condition:

$$
x-x \times 0=y-y \times 0 \rightleftarrows x=y
$$

(On observera que $D$ est toujours normal si $m=n / p=3$.)

En s'occupant d'abord des $x \in D_{i}$, on aura:

$$
\begin{array}{ccc}
x=i, & i+p, \quad i+2 p, \ldots, \quad i+k p, \ldots, \quad i+m p-p . \\
F=j+z_{0} p, \quad j+z_{1} p, \quad j+z_{2} p, \ldots, \quad j+z_{k} p, \ldots, j+z_{m-1} p . \\
x-F=i-j-z_{0} p, i-j-\left(z_{1}-1\right) p, i-j-\left(z_{2}-2\right) p, \ldots, \\
i-j-\left(z_{k}-k\right) p, \ldots, i-j-\left(z_{m-1}-m+1\right) p .
\end{array}
$$


Pour que ces différences soient toutes distinctes deux à deux, il faut que, en posant:

$$
y=0,1,2,3, \ldots, k, \ldots, m-1
$$

et

$$
\rho_{i}(y)=z_{0}, z_{1}, z_{2}, z_{3} \ldots, z_{k} \ldots, z_{m-1},
$$

la fonction $\rho_{i}(y)$ satisfasse à la condition:

$$
y-\rho_{i}(y)=y^{\prime}-\rho_{i}\left(y^{\prime}\right) \rightleftarrows y=y^{\prime}
$$

Cela exprime que $\rho$ définit un quasigroupe d'ordre $m$, automorphe par le groupe cyclique $C_{m}$ et d'ailleurs quelconque.

Si toutes les différences $i-j+\left(k-z_{k}\right) p$ sont distinctes elles seront, à l'ordre près, égales à:

$$
i-j, i-j+p, i-j+2 p, \ldots, i-j+(m-1) p .
$$

En considérant maintenant toutes les valeurs de $x$, il faut exprimer que la suite (6) n'a aucun terme commun avec chacune des suites analogues:

$$
i^{\prime}-j^{\prime}, i^{\prime}-j^{\prime}+p, i^{\prime}-j^{\prime}+2 p, \ldots, i^{\prime}-j^{\prime}+(m-1) p,
$$

où $i, j, i^{\prime}, j^{\prime}<p$.

Or il est facile de voir que, pour que (6) et (7) n'aient aucun terme commun il faut et il suffit que:

$$
i-j \neq i^{\prime}-j^{\prime}
$$

car: [1], si $i-j=i^{\prime}-j^{\prime}$, alors les suites (6) et (7) ont tous leurs termes deux à deux égaux et par conséquent ne sont pas distinctes; [2], si (6) et (7) ont un terme commun, par exemple:

$$
i-j+k p \equiv i^{\prime}-j^{\prime}+k^{\prime} p \quad(\bmod . n)
$$

on en déduira

$$
\left.(i-j)-\left(i^{\prime}-j^{\prime}\right) \equiv\left(k^{\prime}-k\right) p \quad \text { (mod. } m p\right)
$$

ce qui exige

$$
i-j \equiv i^{\prime}-j^{\prime}
$$

et comme $i$ et $j$ sont plus petits que $p$,

$$
i-j=i^{\prime}-j^{\prime} \text {. }
$$

Finalement il faut que la transformation $i \rightarrow j$ satisfasse à

$$
i-j \neq i^{\prime}-j^{\prime}, \quad i \neq i^{\prime},
$$

ou, en posant $j=f(i)$,

$$
i-f(i) \neq i^{\prime}-f\left(i^{\prime}\right), \quad i \neq i^{\prime} .
$$


Donc la fonction $f(i)$ définit encore un quasigroupe, d'ordre $p$, automorphe par le groupe cyclique $C_{p}$. D'ailleurs ce quasigroupe est idempotent car $D_{i} \times D_{i}=D_{i}$.

La réciproque résulte du $\mathrm{N}^{0} 15$ et la dérnière partie du $\mathrm{N}^{0} 16$.

28. Dans les conditions du $N^{\circ} 27$, si $P$ ést l'ordre de l'automorphe de $f(i)$, le nombre des automorphismes de $Q$ est un diviseur de $P m^{g}$ où g désigne comme au $N^{\circ} 25$ le nombre minimum de générateurs indépendants de $Q$.

Tout automorphisme de $Q$ est défini par un ensemble de $p$ substitutions

$$
s_{i}: x \rightarrow x+k p+j-i \quad(k=0,1,2, \ldots, m-1)
$$

où $i \rightarrow j$ est un automorphisme de $f(i)$ et où la substitution $s_{i}$ opère sur un seul diviseur $D_{i}$.

D'autre part, d'après le $N^{0} 24$, tout automorphisme $T$ de $Q$ projette un $D_{i}$ sur un $D_{j}$, c'est-à-dire permute les $D_{i}$ entre eux. $D$ étant normal, $T$ induit sur le quasigroupe quotient $f(i)$ d'ordre $p$ un automorphisme $t$. Et, si $i T=j$, l'automorphisme $T$ induit un isomorphisme de $D_{i}$ sur $D_{j}$.

Le nombre des automorphismes $t$ est au plus égal à celui des automorphismes du quasigroupe $f(i)$, soit $P$.

Comme tous les $D$ sont monogènes, chacun à pour automorphe le seul groupe cyclique d'ordre' $m$.

$$
x \rightarrow x+k p \quad(k=0,1, \ldots, m-1) .
$$

Si $R$ est un isomorphisme de $D_{i}$ avec $D_{j}$,

$$
D_{i} R=D_{j},
$$

comme on a:

$$
D_{i}\left(\begin{array}{c}
x \\
x+k p
\end{array}\right)=D_{i}
$$

et par conséquent

$$
D_{i}\left(\begin{array}{c}
x \\
x+k p
\end{array}\right) R=D_{j}
$$

tous les autres automorphismes seront compris dans la formule:

$$
\left(\begin{array}{c}
x \\
x+k p
\end{array}\right) R \text {. }
$$

Or $D_{j}$ est isomorphe de $D_{i}$ par $x+i \rightarrow x+j$, c'est-à-dire:

$$
\left(\begin{array}{c}
x \\
x+j-i
\end{array}\right) \text {. }
$$

Donc tous les isomorphismes de $D_{i}$ avec $D_{j}$ sont exprimés par les transformations: 
$i, j$ fixes; $k=0,1, \ldots, m-1$.

$$
\begin{aligned}
& S=\left(\begin{array}{c}
x \\
x+k p
\end{array}\right)\left(\begin{array}{c}
x \\
x+j-i
\end{array}\right)=\left(\begin{array}{c}
x \\
x+k p+j-i
\end{array}\right) \\
& 0,1, \ldots, m-1 .
\end{aligned}
$$

Pour chaque $D_{i}$ il existe donc $m$ substitutions, correspondant aux $m$ valeurs de $k$ et projetant $D_{i}$ sur $D_{j}$.

L'automorphisme $T$ de $Q$ induit un isomorphisme de $D_{i}$ avec $D_{j}$ compris dans les $m$ substitutions $S$ ci-dessus. Cela peut être répété pour les $p$ diviseurs $D_{i}$, maís si $Q$ a g générateurs, le choix de k n'est libre que pour ces $g$ générateurs et finalement le nombre total des automorphismes de $Q$ est un diviseur de $P m^{g}$, ordre du groupe défini par les $t$ et par les $S$.

Exemple I.

$$
\begin{aligned}
& \mathrm{F}=(0,3)(1,5)(2,4)(6)(7,8) \\
& p=m=3 ; D_{0}=0,3,6 ; D_{1}=1,4,7 ; D_{2}=2,5,8 \\
& S_{0}=\left(\begin{array}{l}
x \\
x
\end{array}\right) ; \quad S_{1}=\left(\begin{array}{c}
x \\
x+3
\end{array}\right) ; \quad S_{2}=\left(\begin{array}{c}
x \\
x+6
\end{array}\right) \\
& T=(0)(3)(6) \cdot(147) \cdot(285)
\end{aligned}
$$

Exemple II.

$$
\begin{gathered}
f=(0)(1243) ; \rho_{0}=\rho_{2}=\rho_{3}=(02)(1) ; \rho_{1}=(01)(2) \\
F=(0,10)(1,7,9,13)(2,14,8,6)(3,11,12,4)(5)=f+5 \rho_{i}(y) .
\end{gathered}
$$

Ce quasigroupe est engendré par $(0,1)$. Tout automorphisme est défini par les images de 0 et de 1 . L'automorphe de $Q$ est donc d'ordre $9.5 ! / 3 !=180$ ou un diviseur de 180 .

En écrivant $i$ pour $D_{i}$, le quasigroupe quotient devient $f(i)$ lui-même. Son automorphe est du $20^{\text {ème }}$ ordre; il contient $C_{5}$ et le sousgroupe du $4^{\text {ème }}$ ordre $\{(1243)\}$.

\section{Citations}

1. A. A. Albert, Non associative Algebras, Ann. Math. (2), 49 (1942), 696.

2. B. A. Haussmann, O. Ore, Theory of Quasigroùps. Amer. J. Math., 59 (1937), 983.

3. C. Jordan, Traité des substitutions (Paris, 1870).

4. F. Kiokemeister, A theory of normality for quasi-groups, Amer. J. Math., 70 (1948), 100-102.

5. D. Rees, On semi-groups, Proceed. Camb. Phil. Soc., 86 (1940), 387.

6. A. Sade, Quasigroupes (Marseille, 1950).

7. - Contribution à la théorie des quasigroupes, CR Ácad. Sci. Paris, 2.37 (1953), 420-422.

8. G. Scorza, Gruppi Astratti (Roma, 1942).

Lycée Perier, Marseille 